\title{
Progress on the Elucidation of the Antinociceptive Effect of Ginseng and Ginsenosides in Chronic Pain
}

\author{
Mei-Xian $L^{1}{ }^{1}$, Qian-Qi Wei ${ }^{2 *}$ and Huan-Jun $L^{3 *}$ \\ ${ }^{1}$ National and Local Joint Engineering Research Center of Technical Fiber Composites for Safety and Protection, Nantong \\ University, Nantong, China, ${ }^{2}$ Department of Infectious Diseases, General Hospital of Tibet Military Command, Xizang, China, \\ ${ }^{3}$ Institute of Pain Medicine and Special Environmental Medicine, Nantong University, Nantong, China
}

\section{OPEN ACCESS}

Edited by:

Lisa Lione,

University of Hertfordshire,

United Kingdom

Reviewed by:

Vinod Tiwari,

Indian Institute of Technology (BHU),

India

*Correspondence:

Qian-Qi Wei

infection0522@gmail.com

Huan-Jun Lu

huan-jun-/u@hotmail.com

Specialty section:

This article was submitted to

Neuropharmacology,

a section of the journal

Frontiers in Pharmacology

Received: 25 November 2021

Accepted: 26 January 2022

Published: 21 February 2022

Citation:

Li M-X, Wei Q-Q and Lu H-J (2022)

Progress on the Elucidation of the Antinociceptive Effect of Ginseng and

Ginsenosides in Chronic Pain.

Front. Pharmacol. 13:821940.

doi: $10.3389 /$ fphar.2022.821940
Ginseng (Panax ginseng C.A. Meyer) is a traditional Oriental herbal drug widely used in East Asia. Its main active ingredients are ginsenosides whose constituents are known to have various pharmacological activities such as anticancer, antinociception, and neuroprotection. The analgesic effects of ginsenosides, such as Rg1, Rg2, and Rb1, as well as compound $\mathrm{K}$, are well known and the analgesic mechanism of action in inflammatory pain models is thought to be the down regulation of pro-inflammatory cytokine expression (TNF- $\alpha \mathrm{IL}-1 \beta$, and IL-6). Several studies have also demonstrated that ginsenosides regulate neuropathic pain through the modulation of estrogen receptors. Recently, an increasing number of pathways have emerged in relation to the antinociceptive effect of ginseng and ginsenosides. Therefore, this review presents our current understanding of the effectiveness of ginseng in chronic pain and how its active constituents regulate nociceptive responses and their mechanisms of action.

Keywords: chronic pain, ginseng, ginsenosides, antinociception, anti-inflammation

\section{INTRODUCTION}

Pain is a very common concern for patients and has a significant effect upon their quality of life. Specifically, pain, as defined by the International Association for Pain Research, is an unpleasant feeling and emotional experience associated with potential or existing tissue damage. (Treede, 2018). Pain can be divided into acute and chronic pain depending upon its duration. Chronic pain, which is defined as pain lasting for longer than 3 months after the onset of the initial injury or disease, affects the quality of daily living (Mills et al., 2019). Chronic pain is challenging to treat due to the limited efficacy and adverse side effects of therapies. Commonly used analgesics are classified as opioids (cocaine, morphine) and nonsteroidal anti-inflammatory drugs (NSAIDs), which have a good clinical therapeutic effect. However, opioids carry a significant risk of severe side effects including nausea, itching, sedation, respiratory depression, addiction, tolerance and dependence, and even the unacceptably high death rate for repeated dosing of opioids (Lutfy, 2020; Uniyal et al., 2020; Seth et al., 2021). According to the survey, in 2016, around 2.1 million Americans were addicted to opioids, and more than 42,000 patients died due to opioid overdose (Seth et al., 2018). Therefore,

\footnotetext{
Abbreviations: NSAIDs, nonsteroidal anti-inflammatory drugs; 5-HT receptor, 5-hydroxytryptamine receptor; ER, estrogen receptor; JNK, janus kinase; ERK, extracellular signal-regulated kinase; MMPs, matrix metalloproteinases; IBS, irritable bowel syndrome; PNS, peripheral sensitization; CNS, central sensitization; LPS, lipopolysaccharide; IFN- $\gamma$, interferon-gamma; GMCSF, granulocyte-macrophage colony-stimulating factor; MAPKs, mitogen-activated protein kinases; STZ, streptozotocin; PKA, protein kinase A.
} 
clinical guidelines discourage the use of opioids to treat chronic pain (Edelman and Hemmert 2019) and the exploration and discovery of new types of analgesics with little adverse reactions, highly safe and with good selectivity are urgently needed.

Ginseng (Panax ginseng C.A. Meyer) is an herbal plant within the Araliaceae family and belongs to the Panax Genus (Guo et al., 2021). The roots of ginseng contain ginsenoside, a class of steroid glycoside that is responsible for its pharmacological activity. Ginseng has many bio properties such as antioxidant (Chen and Huang, 2019), anti-inflammatory (Im, 2020), analgesia antipruritic (Lee et al., 2018; Luo et al., 2018), and anticancer activities (Zhang J. et al., 2019). Previous phytochemical and pharmacological investigations have also demonstrated antinociceptive effects of ginseng extracts in various pain models including those in relation to the abdomen (Wang et al., 2018), neuropathic pain (Lee et al., 2021b), chronic joint pain (Chen et al., 2014), and incisional pain (Kim et al., 2018). The mechanisms of action suggested to explain these effects include antagonism of adrenergic (Kim et al., 2018), gammaaminobutyric acid (Yin et al., 2011), and opioid receptors (Suh et al., 2000); and regulation of ion channel activity (Lee, Kim, and Shim 2018), mediation of pro-inflammatory cytokine expression (Luo et al., 2018), and immune cell responses (Chen et al., 2014).

In this review, we provide an overview of ginseng and its active constituents and its major therapeutic applications for the treatment of chronic pain (neuropathic pain, inflammatory pain, osteoarthritis pain, abdominal pain, etc.). We discuss neurobiological and pharmacological mechanisms of action of ginseng and ginsenoside's analgesic effects on chronic pain.

\section{CHEMISTRY OF GINSENG AND EFFECT OF GINSENOSIDES}

Ginseng is mainly composed of ginsenosides, polysaccharides, proteins, amino acids, volatile oils, and lignin (Huang et al., 2021). Among these components, ginsenosides have been implicated as the bioactive constituents, which can be divided into protopanaxadiol (with no hydroxyl group at position six), protopanaxatriol (with a hydroxyl group present at position six) (Table 1), oleanolic acid, and ocotillol types, all of which have different effects. Protopanaxadiol mainly includes the ginsenosides $\mathrm{Rb} 1, \mathrm{Rb} 2, \mathrm{Rb} 3, \mathrm{Rc}, \mathrm{Rd}, \mathrm{Rg} 3$, and Rh2 and compound $\mathrm{K}$, whereas protopanaxatriol is mainly composed of the ginsenosides Re, Rg1, Rg2, and Rh1. These types of ginsenosides have different biological activity and different clinical effects, such as regulation of the central nervous system (Chen et al., 2019; Park et al., 2012b; Wu et al., 2018), promoting learning and memory ability (Chen et al., 2018; Jiang et al., 2021), improving heart function (Li X. et al., 2020; Lu M. et al., 2021), hypoglycemic effects (Tabandeh et al., 2015; Meng et al., 2017), improving resistance to stress (Chen et al., 2016; Li c. w. et al., 2020), and modulating the immune response (Park et al., 2015). Based on various clinical studies, the pharmacokinetics of the different types of ginsenosides remain unclear because of their heterogeneous chemical structures (Qi et al., 2011). Here, we highlight their roles and mechanisms of action in the regulation of chronic pain.

\section{GINSENG IN CHRONIC PAIN}

Ginseng has been widely used to modulate various types of chronic pain such as neuropathic pain, inflammatory, and other types of chronic pain. At least 10 of ginseng saponins could inhibit NF- $\kappa B$ transcription factor (Kim et al., 2009) as well as reduce inflammatory NO synthase expression (Huynh et al., 2020), while some ginsenosides have direct effect against neuropathic pain that blocks calcium channels in nociceptive neurons (Mogil et al., 1998). In this section, we will introduce the effects of ginseng and ginsenosides on variety of chronic pain and its mechanism.

\section{Ginseng in Neuropathic Pain}

Neuropathic pain is a type of chronic pain that arises as a direct consequence of a lesion or disease affecting the somatosensory system. Approximately $7-10 \%$ of the population suffers from pain with neuropathic characteristics (Wright and Rizzolo, 2017). The pathogenesis of neuropathic pain is complicated, spinal cord injury (Lee et al., 2021b), immune diseases (Lu H. J. et al., 2021), diabetes (Rosenberger et al., 2020), and cancers (Fallon, 2013) can all cause neuropathic pain. The effect of traditional opioid analgesics and nonsteroidal anti-inflammatory drugs on neuropathic pain are less than ideal. Furthermore, long-term use of opioids are potentially very addictive and can cause other side effects. The bioactive components of ginseng have been reported to possess certain therapeutic effects on neuropathic pain. Recently, Lee et al. (2019) evaluated the effects of ethanol extracted white ginseng (GS-KG9) on streptozotcin (STZ) induced diabetic neuropathic pain in rats. They found that oral administration of GS-KG9 lowered blood glucose levels and inhibited microglial activation in position L4-L5 of the spinal cord in STZ-induced hyperglycemic rats. This analgesic effect of GS-KG9 was found to be mediated by the inhibition of microglial activation through reducing blood glucose levels. GS-KG9 also inhibits hyperexcitability of the spinal cord dorsal horn neurons thus attenuating allodynia and hyperalgesia. Kim et al. (2020) reported that intrathecally administered Korean red ginseng produced a dosedependent antiallodynic effect in a chemotherapy-induced neuropathic pain model. Furthermore, 5-Hydroxytryptamine (5HT) receptor antagonists reversed this effect. Their results suggested that red ginseng is effective against chemotherapyinduced neuropathic pain at the spinal level through mediating 5HT receptor expression. In addition, Suzuki et al. (2017) found that ginseng extracts showed a protective effect against neurite damage induced by oxaliplatin and relieved oxaliplatin induced neuropathic pain in mice. Furthermore, treatment with ginseng extract significantly ameliorated both cold allodynia and mechanical hyperalgesia induced by oxaliplatin, whereas oxaliplatin treatment suppressed neurite outgrowth from primary dorsal root ganglia, and ginseng extract inhibited this suppression. These results suggest that ginseng extract could be an effective agent for the treatment of oxaliplatin-induced neuropathic pain. Lee et al. (2021b) reported that the ginsenoside $\mathrm{Rb} 1$ and compound $\mathrm{K}$ released peripheral and central neuropathic pain through estrogen receptors. Activation of microglia/astrocytes and the expression of inflammatory mediators such as IL- $1 \beta$, iNOS, and Cox- 2 were also significantly inhibited in the L4-L5 spinal cord of central neuropathic rats by oral administration of $\mathrm{Rb} 1$ and compound $\mathrm{K}$. However, the antinociceptive effects of Rb1 
and compound $\mathrm{K}$ were reversed by treatment with estrogen receptor (ER) antagonists. These results indicated that Rb1 and compound $\mathrm{K}$ have potential antinociceptive effects against both peripheral and central neuropathic pain that may be mediated through the estrogen receptor. Li et al. (2019) reported that ginsenoside $\mathrm{Rf}$ can effectively decrease pain hypersensitivity, depression-like behavior and inflammatory reactions in chronic constriction injury induced neuropathic pain model. Chronic treatment of ginsenoside $\mathrm{Rf}$ partially inhibits increase of proinflammatory cytokines in the injured DRG and spinal cord. However, it promotes the IL-10 expression, an anti-inflammatory factor. Those results suggest ginsenoside $\mathrm{Rf}$ alleviates neuropathic pain and its associated depression by restoring the balance between proinflammatory and anti-inflammatory cytokines.

\section{Ginseng in Inflammatory Pain}

In many types of chronic pain, inflammatory pain represents a major component. Inflammatory pain is defined as increased sensitivity caused by an inflammatory response associated with tissue damage (Muley et al., 2016). When inflammatory pain occurs, an innocuous stimulus, which usually would not cause pain can be perceived as painful. This kind of primary hyperalgesia is a direct consequence of peripheral nerve sensitization mediated by inflammatory mediators and other factors such as serotonin, protons (change in $\mathrm{pH}$ ), bradykinin, and cytokines. Recent findings suggest that ginseng has a positive effect on anti-inflammatory pain, and Jang et al. (2016) reported that the ginsenoside Rb1 attenuates acute inflammatory pain by inhibiting neuronal extracellular signalregulated kinase (ERK) phosphorylation via regulation of the Nrf2 and NF- $\kappa$ B pathways. The authors used formalin to induce inflammatory pain in rats and found that intrathecal injection of Rb1 could effectively relieve the formalin-induced acute inflammatory pain response. Furthermore, they also confirmed this analgesic effect was caused by the inhibition of ERK phosphorylation and microglial (astrocyte) activation through regulation of the nuclear factor erythroid 2-related factor 2 nuclear factor kappa B pathway in the spinal cord. Taherianfard and Aalami (2020) also reported that ginseng extract decreased pain sensitivity through regulation of dopamine D2 receptor activity in this formalin model. Furthermore, Nah et al. (2000) investigated the analgesic effect of ginsenosides on a capsaicin-induced inflammatory pain model and found that either intraperitoneally or intrathecally administered ginsenosides suppressed capsaicin-induced nociceptive behavior in a dose dependent manner and that administration of ginsenosides did not significantly affect the motor ability of the animal. Their results suggest that ginsenosides produced antinociceptive effects through their action at spinal or supraspinal sites, not at peripheral nociceptors or opioid receptors.

\section{Ginseng in Other Chronic Pain Conditions}

Ginseng is not only effective in the treatment of neuropathic and inflammatory pain, but also in other types of chronic pain. Osteoarthritis is an age-related degenerative disease, often resulting in chronic joint inflammation and pain. The most common medications used to treat Osteoarthritis are NSAIDs, which increase the risk for gastrointestinal distress (Rannou et al., 2016). Several researchers have also reported ginseng and ginsenosides as having analgesic effects on osteoarthritis induced chronic joint pain. Choi et al. (2013) reported that compound $\mathrm{K}$ decreased the production of matrix metalloproteinases (MMPs) from fibroblast like synoviocytes by inhibiting Janus kinase (JNK) and ERK signaling pathways and Kim et al. (2007) evaluated the antiarthritic effects of ginsenoside $\mathrm{Rb} 1$ in collagen-induced arthritic mice. Their results suggested that oral administration of ginsenoside Rb1 significantly reduced clinical arthritis scores and decreased immune cell infiltration and cartilage destruction. Treatment with ginsenoside Rb1 suppressed TNF- $\alpha$ expression, which was upregulated during inflammatory responses in collagen-induced arthritic mice.

Incisional hernias represent a very common clinical symptom after surgical incision or intraoperative injury and Kim et al. (2018) reported that ginsenoside $\mathrm{Rf}$ had significant antinociceptive and anti-inflammatory effects on an incisional pain model in rats. Ginsenoside Rf increased the mechanical withdrawal threshold significantly, with a curvilinear dose-response curve peaking at $1.5 \mathrm{mg} / \mathrm{kg}$ and the levels of IL- $1 \beta$, IL- 6 , and TNF- $\alpha$ significantly decreased after ginsenoside $\mathrm{Rf}$ treatment. Interestingly, the antinociceptive effect of ginsenoside $\mathrm{Rf}$ was reduced by yohimbine, but potentiated by prazosin and ketanserin, suggesting that adrenergic receptors might be involved in pain regulation by ginsenoside Rf. Irritable bowel syndrome (IBS) is a chronic gastrointestinal disorder associated with abdominal pain and irregular bowel habits such as constipation and diarrhea. Currently, IBS is recognized to be a multifactorial disorder with a heterogeneous and complex pathophysiology. Thus, It is difficult to develop safe and effective therapy (Chey et al., 2015). Ginseng as a traditional health care medicine is also used to treat IBS in Asia. Kim et al. (2005) evaluated the effect of ginsenosides on a rat model of IBS and found that they inhibited 5-HT3A receptor channel activity. Furthermore, because the 5-HT3A receptor is closely related with IBS in enteric nervous system, oral administration of ginsenosides significantly and dose-dependently inhibited acetic acid-induced visceral hypersensitivity. A clinical study reported (Rocha et al., 2018) in Brazil also showed that the dry extract of ginseng could reduce abdominal pain in IBS patients. Clearly, from the above-mentioned pain causes ginseng and ginsenoside have a good therapeutic effect. In addition, many studies have reported that ginseng and ginsenosides also have effects on bone cancer pain, cancer pain, and migraine. As further research and clinical applications are increasing, it is clear that ginseng shows great potential as a future therapy for the treatment of chronic pain, or as an adjunct treatment for multiple pain types.

\section{MECHANISM OF ACTION, TARGET AND SIGNALING PATHWAYS}

Chronic pain is maintained by both central sensitization and peripheral sensitization, where neuronal responsiveness increases, thus presenting the phenomenon of synaptic plasticity (Figure 1). With the advent of noxious stimuli, neuroinflammation is triggered causing the activation of glial cells in dorsal root ganglia, spinal cord, and brain which drives the production of pro-inflammatory cytokines and 


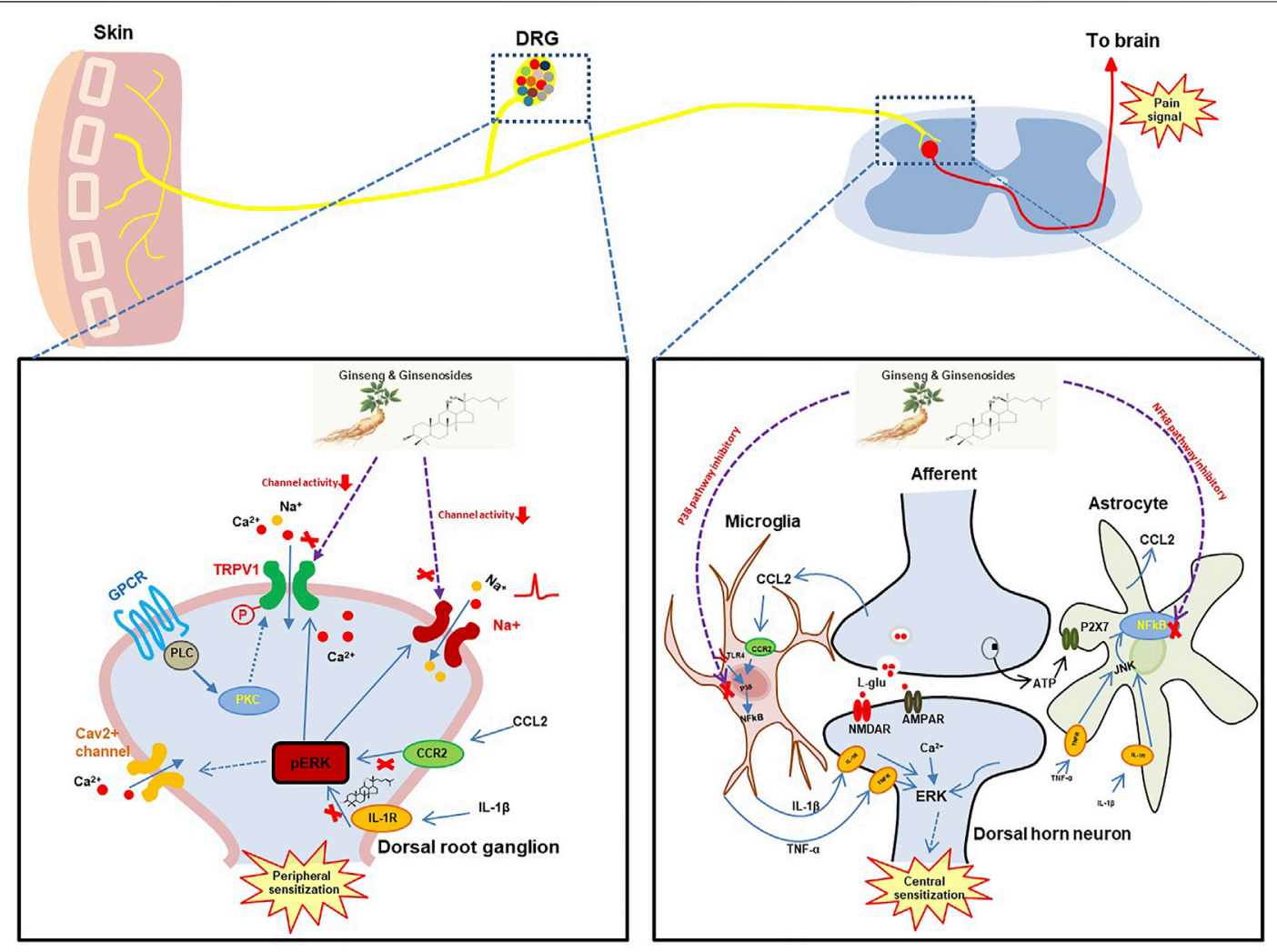

FIGURE 1 | The hypothetical analgesic action mechanisms of ginseng and ginsenosides. DRG, dorsal root ganglion; TRPV1, transient receptor potential cation channel subfamily V member 1; GPCR, G protein-coupled receptor; PLC, phospholipase C; PKC, protein kinase C; CCR2, C-C motif chemokine receptor 2; CCL2, C-C motif chemokine ligand 2; TLR4, Toll-like receptor 4; JUK, Janus kinase; NMDAR, N-methyl-D-aspartate receptor;AMPAR, $\alpha$-amino-3-hydroxy-5-methyl-4isoxazolepropionic acid receptor.

chemokines in the PNS (peripheral sensitization) and CNS (central sensitization) (Ji et al., 2018; Matsuda et al., 2019). Studies have found that ginseng not only plays an important role in the anti-inflammatory process but reduces hypersensitivity in neurons. It also helps to enhance inhibitory circuitry in pain transduction and bridge the gap between microglia and neurons by regulating inflammatory molecules. Hence now we will focus on the antinociceptive mechanism of ginseng on specific targets.

\section{Microglia}

Microglia are a group of resident macrophages originating from the yolk sac and belong to mononuclear phagocyte system, where they play a role in immune surveillance in the CNS (Suzumura 2013; Nayak et al., 2014). Microglia remain inactive under normal physiological states, but then become activated during injury or during a pathogen invasion. Evidence suggests that microglia represent key players in the development and maintenance of chronic pain (Ji et al., 2013). Depending upon the type of stimulus, normally lipopolysaccharide (LPS) or IL-4, microglia can be classified into classical activation state M1 as a pro-inflammatory state which also has neurotoxic effects, as well as an alternative activation state M2 involved in neuroprotective process (Hernandez Baltazar et al., 2020; Orihuela et al., 2016; Tu et al., 2021).
Though the activation of microglia can be beneficial during the recognition of pathogen or abnormal protein aggregation, inducing cellular phagocytosis, the persistent activation of microglia generates hyper-neuroinflammation which then facilitates hyperexcitability of pain signaling (Tiwari et al., 2014).

The multiple constituents of ginseng including the ginsenosides $\mathrm{Rb} 1, \mathrm{Rb} 2, \mathrm{Rg} 1, \mathrm{Rg} 3, \mathrm{Re}, \mathrm{Rh} 1, \mathrm{Rh} 2$, and its metabolite compound $\mathrm{K}$ inhibit the activation of microglia, thereby ameliorating inflammatory cellular pathways such as NF-kB signaling (Jung et al., 2010; Park et al., 2012; Park et al., 2012a; Shi et al., 2019; Wu et al., 2007; Xu et al., 2020; Yao et al., 2019). For example Rg1 can attenuate the expression of iNOS, COX-2, TNF- $\alpha$, IL- $1 \beta$, and NF- $\mathrm{kB}$ in LPS-induced BV-2 microglial cells via the activation of the PLC- $\gamma 1$ signaling pathway, and this involves the phosphorylation of IkB- $\alpha$, CREB, ERK1/2, JNK, and p38 MAPK (Zong et al., 2012). Furthermore, the ginsenoside Rg1 can mediate the expression of neuroinflammatory biomarkers and inhibit chemotherapy-induced microglial polarization from M2 to M1 phenotypes (Shi et al., 2019). A further mechanism involving the ER has been reported in a recent study. Administration of Rb1 has been found to alleviate allodynia and hyperalgesia in central neuropathic pain-induced rats by inhibition of microglia in the L4-L5 spinal cord. These antinociceptive effects of Rbl can be reversed by ER antagonist, indicating that the antinociceptive mechanism of action of ginseng is clearly complex in microglia and may involve several intracellular 
TABLE 1 | Classification of ginsenosides and their effects.

\begin{tabular}{|c|c|c|c|c|}
\hline Type & Chemical formula & Ginsenoside & Effects & References \\
\hline $\begin{array}{l}\text { Protopanaxadiol } \\
\text { type }\end{array}$ & & $\begin{array}{l}\text { Ra1, Ra2, Ra3, Rb1, } \\
\text { Rb2, Rb3, Rc, Rd, Rg3, } \\
\text { Rh2, F2, R1 }\end{array}$ & $\begin{array}{l}\text { Diverse anticancer activities; } \\
\text { Regulating central nervous system; } \\
\text { Improving learning and memory; } \\
\text { Analgesic effect }\end{array}$ & $\begin{array}{l}\text { Chen et al. (2018), Jiang et al. (2021), } \\
\text { Kim et al. (2018), Park et al. (2012b), } \\
\text { Sun et al. (2019), Wang et al. (2021), } \\
\text { Wu et al. (2018) }\end{array}$ \\
\hline $\begin{array}{l}\text { Protopanaxatriol } \\
\text { type }\end{array}$ & & $\begin{array}{l}\text { Re, Rf, Rg1, Rg2, Rh1, } \\
\text { R1, R2, F1 }\end{array}$ & $\begin{array}{l}\text { Improving heart function; Inhibiting the } \\
\text { growth of tumor cells; Anti- } \\
\text { inflammatory } \\
\text { Relieve neuropathic pain }\end{array}$ & $\begin{array}{l}\text { Huang et al. (2019), Kim et al. (2012), Li } \\
\text { C. W. et al. (2020), Li et al. (2019), Lu } \\
\text { et al. (2021), Zhang Q.-L. et al. (2019) }\end{array}$ \\
\hline $\begin{array}{l}\text { Oleanolic acid } \\
\text { type }\end{array}$ & & $\mathrm{RO}, \mathrm{Rh} 3, \mathrm{Ri}$ & $\begin{array}{l}\text { Anti-inflammatory; Anti-thrombotic } \\
\text { effect }\end{array}$ & $\begin{array}{l}\text { He et al. (2013), Hu et al. (2008), Lee } \\
\text { et al. (2015), Lin et al. (2016) }\end{array}$ \\
\hline Ocotillol type & & $\mathrm{R} 2, \mathrm{Rg} 5$ & $\begin{array}{l}\text { Inhibiting the growth of tumor cells; } \\
\text { Proliferation and apoptosis of cancer } \\
\text { cells }\end{array}$ & Dong et al. (2019), Jeong et al. (2015) \\
\hline
\end{tabular}

R1: Glc-Glc- or G/C- or H.

R2: Rha-Glc-O- or Glc-Glc-O- or Glc-O- or $\mathrm{H}$ or $\mathrm{OH}$.

R3: G/C-G/C- or A-G/c- or G/c- or $\mathrm{H}$.

R4: Glc-.

pathways and therefore further investigation is needed its full elucidation (Lee et al., 2021a).

\section{Neurons}

The crosstalk between neurons and microglia involving cytokines and chemokines represents one aspect of pin sensitization (Figure 1). However, multiple ion channels such as voltagegated $\mathrm{Na}^{+}$channels and voltage-gated $\mathrm{Ca}^{2+}$ channels localized in primary sensory neurons remain a key component in the perception of pain associated with the pathogenesis of chronic neuropathic and inflammatory pain conditions (Lee et al., 2021a).

A polyacetylenic compound isolated from ginseng extract has been found to inhibit $\mathrm{Na}^{+}$currents and can accelerate their inactivation, thus producing a hyperpolarizing shift of the steadystate inactivation curve in acutely dissociated rat dorsal root ganglion (DRG) neurons (Choi et al., 2008). Similarly, application of ginseng total saponins, in particular ginsenoside Rg3, suppresses high-voltageactivated $\mathrm{Ca}^{2+}$ channel currents via pertussis toxin-sensitive G-protein pathways similar to that seen for $\mu$-opioid receptors (Rhim et al., 2002). In addition, red ginseng extract has an inhibitory effect on histamine-induced itch sensation by inhibition of the H1R/TRPV1 ion channel present in DRG neurons (Jang et al., 2015). Noteworthy, TRPV1 is a non-selective cation channel highly expressed in DRG neurons and the spinal cord, believed to serve as a viable target in the regulation of chemotherapy-induced neuropathic pain (Akhilesh et al., 2022). Rg1 blocks intracellular calcium by both capsaicin and proton activation in a TRPV1-dependent manner indicating its potential as an antagonist of TRPV1 (Huang et al., 2012). However, it seems that the antinociceptive effect of ginseng is more potent in the central than peripheral nervous system. Intrathecal administration of ginsenosides was found to intracerebroventricularly suppress capsaicin-induced pain-related behavior in mice rather than when administered 
subcutaneously. Ginsenosides can also inhibit excitatory neurotransmitter release from primary sensory nerve terminals following capsaicin injection independently of opioid receptors (Nah et al., 2000). The same group also finds that ginsenoside Rc enhanced capsaicin-induced inward currents in Xenopus oocytes expressing the vanilloid receptor in a concentration-dependent and reversible manner. This suggests a different channel is utilized by ginsenosides to regulate capsaicin-induced pain in DRG neurons (Jung et al., 2001).

A recent study has reported that ginseng influences the release of dopamine through the cholinergic system, and directly affects dopamine D2 receptors to exert its analgesic effect. Application of ginseng extract alone or with D2 receptor agonists synergistically has an obvious analgesic effect with no additive effect (Taherianfard and Aalami 2020). Taken together these findings indicate that the antinociceptive mechanism of action of ginseng between neurons could be extensive.

The N-methyl-D-aspartate (NMDA) receptors are excitatory glutamate receptors and widely expressed in the pain transmission pathway whose hyperactivity fires pain signal projecting to the supraspinal structures of the CNS, making it a progress to central sensitization (Uniyal et al., 2021). The weak efficacy of traditional NMDA antagonists such as ketamine and memantine applicated in pain management suggests the urgent needs for development of new NMDA receptor antagonists for analgesia (Kreutzwiser and Tawfic, 2019; Uniyal et al., 2021). Intraperitoneal injection of ginseng total saponins in hyperalgesia model significantly increases mechanical withdrawal threshold, and this antinociception of ginseng total saponins is reserved by additional injection of NMDA (Kim et al., 2014). Ginsenoside Rg3 and Rk1 effectively antagonize NMDA receptors in rat neurons through respective mechanisms, indicating the potency of ginsenosides as NMDA antagonists in pain management (Ryoo et al., 2020).

\section{Other Potential Targets}

When compared to microglia, little work has been reported concerning the effects of ginsenosides in other glial cells including astrocytes and oligodendrocytes, both important in central sensitization (Donnelly et al., 2020; Kwon and Koh, 2020). Based on current findings, ginseng functions to protect astrocytes from hypoxia or oxidant stressors (Naval et al., 2007; Choi et al., 2019), facilitating clearance of extracellular glutamate (Zhang et al., 2013), inducing astroglial autophagy (Rahman et al., 2020) and inhibiting their activation during ischemia and mild stress (Fan et al., 2018; Liu et al., 2020). Gintonin facilitates late differentiation of oligodendrocytes in primary oligodendrocyte precursor cells, suggesting a potential new treatment for demyelinating diseases (Mijan et al., 2019). Schwann cells are important glial cells in the PNS, and provide neurotropic support for peripheral nerves after injury and can interact with nociceptive neurons to regulate different pain conditions (Wei et al., 2019). Ginseng and Rg1 enhance proliferation of RSC96 Schwann cells in a dose-dependent manner by increasing the phosphorylation of ERK, NH2-terminal JNK, and p38, which are sub-families of mitogen-activated protein kinases (MAPKs). In addition, ginseng and Rg1 also stimulate the FGF-2-uPA-MMP-9 migration pathway to enhance the migration of RSC96 Schwann cells (Lu et al., 2009). Similarly, Rb1 and Rg1 promotes secretion of nerve growth factor and brain-derived neurotropic factor in cultured Schwann cells mainly through the protein kinase A (PKA) pathway (Liang et al., 2010). Wang et al. reported that $\operatorname{Re}$ also promotes Schwann cell proliferation, differentiation, and migration in vitro via activating ERK- and JNK-signaling (Wang et al., 2015). As mentioned above, like microglia, macrophages have proinflammatory M1-like and anti-inflammatory M2-like phenotypes, and their cytokine pattern of release can influence pain transduction and neuronal conduction via modulation of ion channels (Ji et al., 2016). Ginseng promotes the polarization of M2 to M1 phenotype and increases the production of immunomodulators such as TNF- $\alpha$, IL$1 \beta$, IL-6, NO, iNOS, and COX-2, which causes enhanced macrophagic phagocytosis of bacteria whose components such as LPS produce pain hypersensitivity in nociceptors (Cao et al., 2019; Xin et al., 2019; Um et al., 2020). From these findings further research remains necessary into the direct/indirect analgesic role of ginseng and its ginsenosides in pain modulation and in the intricate pain sensitivity loop between glial cells and immune cells both in the PNS and CNS.

\section{CONCLUSION}

Chronic pain is typically characterized by peripheral mechanical allodynia and heat hyperalgesia. Opioids and NSAIDs are wellestablished pharmacological therapies for the treatment of chronic pain. However, these drugs have not always been effective against chronic pain and have some side effects because of their binding to off-site targets. The potential analgesic effects of ginsenosides on chronic pain are very clear and effective. Recent studies have focused upon the effects of natural anti-inflammatory drugs such as ginsenosides on chronic pain treatment, largely due to their relative safety, reliability, and affordability. The molecular and cellular mechanisms of action of ginseng and its bioactive constituents the ginsenosides include the modulation of neurotransmitter function in both peripheral and central systems, inhibition of inflammatory cytokine expression, modulation of ion channel activity in DRG and spinal cord neurons, regulation of the TLR $4 / \mathrm{NF}-\mathrm{\kappa B}$ signal transduction axis, and anti-inflammatory effects. This review has provided a theoretical basis and clinical application for the treatment of chronic pain based on Panax ginseng. Hence, ginsenosides have great potential for future pain treatment or as an adjuvant for pain therapy in multiple pain types.

\section{AUTHOR CONTRIBUTIONS}

M-XL, Q-QW and H-JL designed and wrote this review manuscript.

\section{FUNDING}

This study was supported by National Natural Science Foundation of China (NSFC 32100806) and Natural Science Foundation of the Higher Education Institutions of Jiangsu Province (Grant No. 21KJB310010). 


\section{REFERENCES}

Akhilesh, Uniyal, A., Uniyal, A., Gadepalli, A., Tiwari, V., Allani, M., et al. (2022). Unlocking the Potential of TRPV1 Based siRNA Therapeutics for the Treatment of Chemotherapy-Induced Neuropathic Pain. Life Sci. 288, 120187. doi:10.1016/j.lfs.2021.120187

Cao, M., Yan, H., Han, X., Weng, L., Wei, Q., Sun, X., et al. (2019). Ginseng-derived Nanoparticles Alter Macrophage Polarization to Inhibit Melanoma Growth. J. Immunother. Cancer 7, 326. doi:10.1186/s40425-019-0817-4

Chen, F., and Huang, G. (2019). Antioxidant Activity of Polysaccharides from Different Sources of Ginseng. Int. J. Biol. Macromol 125, 906-908. doi:10.1016/j. ijbiomac.2018.12.134

Chen, J., Wu, H., Wang, Q., Chang, Y., Liu, K., Song, S., et al. (2014). Ginsenoside Metabolite Compound K Alleviates Adjuvant-Induced Arthritis by Suppressing T Cell Activation. Inflammation 37, 1608-1615. doi:10.1007/s10753-014-9887-0

Chen, J., Zhang, X., Liu, X., Zhang, C., Shang, W., Xue, J., et al. (2019). Ginsenoside Rg1 Promotes Cerebral Angiogenesis via the PI3K/Akt/mTOR Signaling Pathway in Ischemic Mice. Eur. J. Pharmacol. 856, 172418. doi:10.1016/j.ejphar.2019.172418

Chen, L., Yao, H., Chen, X., Wang, Z., Xiang, Y., Xia, J., et al. (2018). Ginsenoside Rg1 Decreases Oxidative Stress and Down-Regulates Akt/mTOR Signalling to Attenuate Cognitive Impairment in Mice and Senescence of Neural Stem Cells Induced by D-Galactose. Neurochem. Res. 43, 430-440. doi:10.1007/s11064-017-2438-y

Chen, W., Wang, J., Luo, Y., Wang, T., Li, X., Li, A., et al. (2016). Ginsenoside Rb1 and Compound K Improve Insulin Signaling and Inhibit ER Stress-Associated NLRP3 Inflammasome Activation in Adipose Tissue. J. Ginseng Res. 40, 351-358. doi:10.1016/j.jgr.2015.11.002

Chey, W. D., Kurlander, J., and Eswaran, S. (2015). Irritable Bowel Syndrome. Jama 313, 949-958. doi:10.1001/jama.2015.0954

Choi, S. H., Kim, H. J., Cho, H. J., Park, S. D., Lee, N. E., Hwang, S. H., et al. (2019). Gintonin, a Ginseng-Derived Exogenous Lysophosphatidic Acid Receptor Ligand, Protects Astrocytes from Hypoxic and Re-oxygenation Stresses through Stimulation of Astrocytic Glycogenolysis. Mol. Neurobiol. 56, 3280-3294. doi:10.1007/s12035-018-1308-1

Choi, S. J., Kim, T. H., Shin, Y. K., Lee, C. S., Park, M., Lee, H. S., et al. (2008). Effects of a Polyacetylene from Panax Ginseng on Na+ Currents in Rat Dorsal Root Ganglion Neurons. Brain Res. 1191, 75-83. doi:10.1016/j.brainres.2007.11.047

Choi, Y. S., Kang, E. H., Lee, E. Y., Gong, H. S., Kang, H. S., Shin, K., et al. (2013). Joint-protective Effects of Compound K, a Major Ginsenoside Metabolite, in Rheumatoid Arthritis: In Vitro Evidence. Rheumatol. Int. 33, 1981-1990. doi:10.1007/s00296-013-2664-9

Dong, Y., Fu, R., Yang, J., Ma, P., Liang, L., Mi, Y., et al. (2019). Folic Acid-Modified Ginsenoside Rg5-Loaded Bovine Serum Albumin Nanoparticles for Targeted Cancer Therapy In Vitro and In Vivo. Int. J. Nanomedicine 14, 6971-6988. doi:10.2147/IJN.S210882

Donnelly, C. R., Andriessen, A. S., Chen, G., Wang, K., Jiang, C., Maixner, W., et al. (2020). Central Nervous System Targets: Glial Cell Mechanisms in Chronic Pain. Neurotherapeutics 17, 846-860. doi:10.1007/s13311-020-00905-7

Edelman, L. S., and Hemmert, R. (2019). Opioid Use in Long-Term Care: Guidelines and Policy Recommendations. J. Gerontol. Nurs. 45, 5-10. doi:10.3928/00989134-20190813-02

Fallon, M. T. (2013). Neuropathic Pain in Cancer. Br. J. Anaesth. 111, 105-111. doi:10.1093/bja/aet208

Fan, C., Song, Q., Wang, P., Li, Y., Yang, M., and Yu, S. Y. (2018). Neuroprotective Effects of Ginsenoside-Rg1 against Depression-like Behaviors via Suppressing Glial Activation, Synaptic Deficits, and Neuronal Apoptosis in Rats. Front. Immunol. 9, 2889. doi:10.3389/fimmu.2018.02889

Guo, M., Shao, S., Wang, D., Zhao, D., Wang, M., and Meyer', C. A. (2021). Recent Progress in Polysaccharides from Panax Ginseng C. A. Meyer. Food Funct., 12, 494-518. doi:10.1039/d0fo01896aRecent Progress in Polysaccharides from Panax Ginseng C. A. MeyerFood Funct.

He, Z.-M., Wang, X.-H., Li, G.-F., Sun, J.-M., Yang, H., Gao, Y.-G., et al. (2013). Screening and Structures Characterization Ofa-Glucosidase Inhibitors from Total Saponins of Ginseng Stems and Leaves by Ultrafiltration LC-MSn. Chin. J. Anal. Chem. (Chinese Version) 41, 1694-1698. doi:10.3724/sp.j.1096.2013.30532

Hernandez Baltazar, D., Nadella, R., Barrientos Bonilla, A., Flores Martínez, Y., Olguín, A., Heman Bozadas, P., et al. (2020). Does Lipopolysaccharide-Based Neuroinflammation Induce Microglia Polarization. Folia Neuropathol. 58, 113-122. doi:10.5114/fn.2020.96755
Hu, P., Luo, G. A., Wang, Q., Zhao, Z. Z., Wang, W., and Jiang, Z. H. (2008). The Retention Behavior of Ginsenosides in HPLC and its Application to Quality Assessment of Radix Ginseng. Arch. Pharm. Res. 31, 1265-1273. doi:10.1007/ s12272-001-2105-2

Huang, J., Ding, L., Shi, D., Hu, J. H., Zhu, Q. G., Gao, S., et al. (2012). Transient Receptor Potential Vanilloid-1 Participates in the Inhibitory Effect of Ginsenoside Rg1 on Capsaicin-Induced Interleukin-8 and Prostaglandin E2 Production in HaCaT Cells. J. Pharm. Pharmacol. 64, 252-258. doi:10.1111/j.2042-7158.2011.01392.x

Huang, L. L., Tang, M., Du, Q. Q., Liu, C. X., Yan, C., Yang, J. L., et al. (2019). The Effects and Mechanisms of a Biosynthetic Ginsenoside 3 $\beta, 12 \beta-D i-O-G l c-P P D$ on Non-small Cell Lung Cancer. Onco Targets Ther. 12, 7375-7385. doi:10. 2147/OTT.S217039

Huang, L., Ren, C., Li, H. J., and Wu, Y. C. (2021). Recent Progress on Processing Technologies, Chemical Components, and Bioactivities of Chinese Red Ginseng. American Red Ginseng, and Korean Red Ginseng', Food and Bioprocess Technology.

Huynh, D. T. N., Baek, N., Sim, S., Myung, C. S., and Heo, K. S. (2020). Minor Ginsenoside Rg2 and Rh1 Attenuates LPS-Induced Acute Liver and Kidney Damages via Downregulating Activation of TLR4-STAT1 and Inflammatory Cytokine Production in Macrophages. Int. J. Mol. Sci. 21, 6656. doi:10.3390/ijms21186656

Im, D. S. (2020). 'Pro-Resolving Effect of Ginsenosides as an Anti-inflammatory Mechanism of Panax Ginseng, 10. doi:10.3390/biom10030444 Biomolecules

Jang, M., Lee, M. J., Choi, J. H., Kim, E. J., Nah, S. Y., Kim, H. J., et al. (2016). Ginsenoside Rb1 Attenuates Acute Inflammatory Nociception by Inhibition of Neuronal ERK Phosphorylation by Regulation of the Nrf2 and NF-Kb Pathways. J. Pain 17, 282-297. doi:10.1016/j.jpain.2015.10.007

Jang, Y., Lee, W. J., Hong, G. S., and Shim, W. S. (2015). Red Ginseng Extract Blocks Histamine-dependent Itch by Inhibition of H1R/TRPV1 Pathway in Sensory Neurons. J. Ginseng Res. 39, 257-264. doi:10.1016/j.jgr.2015.01.004

Jeong, J. J., Van Le, T. H., Lee, S. Y., Eun, S. H., Nguyen, M. D., Park, J. H., et al. (2015). Anti-inflammatory Effects of Vina-Ginsenoside R2 and Majonoside R2 Isolated from Panax Vietnamensis and Their Metabolites in Lipopolysaccharide-Stimulated Macrophages. Int. Immunopharmacol 28, 700-706. doi:10.1016/j.intimp.2015.07.025

Ji, R. R., Berta, T., and Nedergaard, M. (2013). Glia and Pain: Is Chronic Pain a Gliopathy. Pain 154 Suppl 1 (Suppl. 1), S10-S28. doi:10.1016/j.pain.2013.06.022

Ji, R. R., Chamessian, A., and Zhang, Y. Q. (2016). Pain Regulation by Non-neuronal Cells and Inflammation. Science 354, 572-577. doi:10.1126/science.aaf8924

Ji, R. R., Nackley, A., Huh, Y., Terrando, N., and Maixner, W. (2018). Neuroinflammation and Central Sensitization in Chronic and Widespread Pain. Anesthesiology 129, 343-366. doi:10.1097/ALN.0000000000002130

Jiang, N., Wang, K., Zhang, Y., Huang, H., Lv, J. W., Wang, Q., et al. (2021). Protective Effect of Ginsenoside Rb1 against Chronic Restraint Stress (CRS)induced Memory Impairments in Rats. Behav. Brain Res. 405, 113146. doi:10. 1016/j.bbr.2021.113146

Jung, J. S., Shin, J. A., Park, E. M., Lee, J. E., Kang, Y. S., Min, S. W., et al. (2010). Anti-inflammatory Mechanism of Ginsenoside Rh1 in LipopolysaccharideStimulated Microglia: Critical Role of the Protein Kinase A Pathway and Hemeoxygenase-1 Expression. J. Neurochem. 115, 1668-1680. doi:10.1111/j. 1471-4159.2010.07075.x

Jung, S. Y., Choi, S., Ko, Y. S., Park, C. S., Oh, S., Koh, S. R., et al. (2001). Effects of Ginsenosides on Vanilloid Receptor (VR1) Channels Expressed in Xenopus Oocytes. Mol. Cell 12, 342-346.

Kim, H. A., Kim, S., Chang, S. H., Hwang, H. J., and Choi, Y. N. (2007). Antiarthritic Effect of Ginsenoside Rb1 on Collagen Induced Arthritis in Mice. Int. Immunopharmacol 7, 1286-1291. doi:10.1016/j.intimp.2007.05.006

Kim, J. H., Lee, J. H., Jeong, S. M., Lee, B. H., Yoon, I. S., Lee, J. H., et al. (2005). Effect of Ginseng Saponins on a Rat Visceral Hypersensitivity Model. Biol. Pharm. Bull. 28, 2120-2124. doi:10.1248/bpb.28.2120

Kim, J. W., Jung, S. Y., Kwon, Y. H., Lee, J. H., Lee, Y. M., Lee, B. Y., et al. (2012). Ginsenoside Rg3 Attenuates Tumor Angiogenesis via Inhibiting Bioactivities of Endothelial Progenitor Cells. Cancer Biol. Ther. 13, 504-515. doi:10.4161/cbt.19599

Kim, M. K., Kang, H., Baek, C. W., Jung, Y. H., Woo, Y. C., Choi, G. J., et al. (2018). Antinociceptive and Anti-inflammatory Effects of Ginsenoside Rf in a Rat Model of Incisional Pain. J. Ginseng Res. 42, 183-191. doi:10.1016/j.jgr.2017.02.005

Kim, S. M., Lee, S. Y., Yuk, D. Y., Moon, D. C., Choi, S. S., Kim, Y., et al. (2009). Inhibition of NF-kappaB by Ginsenoside Rg3 Enhances the Susceptibility of colon Cancer Cells to Docetaxel. Arch. Pharm. Res. 32, 755-765. doi:10.1007/ s12272-009-1515-4 
Kim, W. J., Kang, H., Kim, J. E., Choi, G. J., Shin, H. Y., Baek, C. W., et al. (2014). Effect of Intraperitoneal Administered Ginseng Total Saponins on Hyperalgesia Induced by Repeated Intramuscular Injection of Acidic saline in Rats. J. Med. Food 17, 657-662. doi:10.1089/jmf.2013.2952

Kim, Y. O., Song, J. A., Kim, W. M., and Yoon, M. H. (2020). Antiallodynic Effect of Intrathecal Korean Red Ginseng in Cisplatin-Induced Neuropathic Pain Rats. Pharmacology 105, 173-180. doi:10.1159/000503259

Kreutzwiser, D., and Tawfic, Q. A. (2019). Expanding Role of NMDA Receptor Antagonists in the Management of Pain. CNS Drugs 33, 347-374. doi:10.1007/ s40263-019-00618-2

Kwon, H. S., and Koh, S. H. (2020). Neuroinflammation in Neurodegenerative Disorders: the Roles of Microglia and Astrocytes. Transl Neurodegener 9, 42. doi:10.1186/s40035-020-00221-2

Lee, J. Y., Choi, H. Y., Park, C. S., Kim, D. H., and Yune, T. Y. (2021b). 'Total Saponin Extract, Ginsenoside Rb1, and Compound K Alleviate Peripheral and central Neuropathic Pain through Estrogen Receptors on Rats. Phytotherapy Res. 35, 2119-2132.

Lee, J. Y., Choi, H. Y., Park, C. S., Kim, D. H., and Yune, T. Y. (2021a). Total Saponin Extract, Ginsenoside Rb1, and Compound K Alleviate Peripheral and central Neuropathic Pain through Estrogen Receptors on Rats. Phytother Res. 35, 2119-2132. doi:10.1002/ptr.6960

Lee, J. Y., Choi, H. Y., Park, C. S., Pyo, M. K., Yune, T. Y., Kim, G. W., et al. (2019). GS-KG9 Ameliorates Diabetic Neuropathic Pain Induced by Streptozotocin in Rats. J. Ginseng Res. 43, 58-67. doi:10.1016/j.jgr.2017.08.004

Lee, W. J., Kim, Y. S., and Shim, W. S. (2018). Korean Red Ginseng Extract and Ginsenoside Rg3 Have Anti-pruritic Effects on Chloroquine-Induced Itch by Inhibition of MrgprA3/TRPA1-Mediated Pathway. J. Ginseng Res. 42, 470-475. doi:10.1016/j.jgr.2017.05.004

Lee, Y. Y., Park, J. S., Lee, E. J., Lee, S. Y., Kim, D. H., Kang, J. L., et al. (2015). Antiinflammatory Mechanism of Ginseng Saponin Metabolite Rh3 in Lipopolysaccharide-Stimulated Microglia: Critical Role of 5'-adenosine Monophosphate-Activated Protein Kinase Signaling Pathway. J. Agric. Food Chem. 63, 3472-3480. doi:10.1021/jf506110y

Li, C. W., Deng, M. Z., Gao, Z. J., Dang, Y. Y., Zheng, G. D., Yang, X. J., et al. (2020a). Effects of Compound K, a Metabolite of Ginsenosides, on Memory and Cognitive Dysfunction in Db/db Mice Involve the Inhibition of ER Stress and the NLRP3 Inflammasome Pathway. Food Funct. 11, 4416-4427. doi:10.1039/c9fo02602a

Li, X., Xiang, N., and Wang, Z. (2020b). Ginsenoside Rg2 Attenuates Myocardial Fibrosis and Improves Cardiac Function after Myocardial Infarction via AKT Signaling Pathway. Biosci. Biotechnol. Biochem. 84, 2199-2206. doi:10.1080/ 09168451.2020.1793292

Li, Y., Chen, C., Li, S., and Jiang, C. (2019). Ginsenoside Rf Relieves Mechanical Hypersensitivity, Depression-like Behavior, and Inflammatory Reactions in Chronic Constriction Injury Rats. Phytother Res. 33, 1095-1103. doi:10.1002/ptr.6303

Liang, W., Ge, S., Yang, L., Yang, M., Ye, Z., Yan, M., et al. (2010). Ginsenosides Rbl and Rg1 Promote Proliferation and Expression of Neurotrophic Factors in Primary Schwann Cell Cultures. Brain Res. 1357, 19-25. doi:10.1016/j.brainres.2010.07.091

Lin, Y. P., Zhang, M. P., Wang, K. Y., Sun, C. Y., and Wang, Y. (2016). Research Achievements on Ginsenosides Biosynthesis from Panax Ginseng. Zhongguo Zhong Yao Za Zhi 41, 4292-4302. doi:10.4268/cjcmm20162302

Liu, L., Vollmer, M. K., Kelly, M. G., Fernandez, V. M., Fernandez, T. G., Kim, H., et al. (2020). Reactive Gliosis Contributes to Nrf2-dependent Neuroprotection by Pretreatment with Dimethyl Fumarate or Korean Red Ginseng against Hypoxic-Ischemia: Focus on Hippocampal Injury. Mol. Neurobiol. 57, 105-117. doi:10.1007/s12035-019-01760-0

Lu, H. J., Fu, Y. Y., Wei, Q. Q., and Zhang, Z. J. (2021). Neuroinflammation in HIVRelated Neuropathic Pain. Front. Pharmacol. 12, 653852. doi:10.3389/fphar. 2021.653852

Lu, M. C., Lai, T. Y., Hwang, J. M., Chen, H. T., Chang, S. H., Tsai, F. J., et al. (2009). Proliferation- and Migration-Enhancing Effects of Ginseng and Ginsenoside Rg1 through IGF-I- and FGF-2-Signaling Pathways on RSC96 Schwann Cells. Cell Biochem Funct 27, 186-192. doi:10.1002/cbf.1554

Lu, M., Wang, J., Sun, Y., Li, C., Sun, T. R., Hou, X. W., et al. (2021). Ginsenoside Rg1 Attenuates Mechanical Stress-Induced Cardiac Injury via Calcium Sensing Receptor-Related Pathway. J. Ginseng Res. 45, 683-694. doi:10.1016/j.jgr.2021.03.006

Luo, H. M., Zhu, D. F., Wang, Y., Chen, Y. H., Jiang, R. Z., Yu, P., et al. (2018). doi:10.3390/molecules23061325 Study on the Structure of Ginseng Glycopeptides with Anti-inflammatory and Analgesic Activity, Molecules, 23.
Lutfy, K. (2020). Opioid Crisis-An Emphasis on Fentanyl Analogs. Brain Sci. 10, 485. doi:10.3390/brainsci10080485

Matsuda, M., Huh, Y., and Ji, R. R. (2019). Roles of Inflammation, Neurogenic Inflammation, and Neuroinflammation in Pain. J. Anesth. 33, 131-139. doi:10. 1007/s00540-018-2579-4

Meng, F., Su, X., Li, W., and Zheng, Y. (2017). Ginsenoside Rb3 Strengthens the Hypoglycemic Effect through AMPK for Inhibition of Hepatic Gluconeogenesis. Exp. Ther. Med. 13, 2551-2557. doi:10.3892/etm. 2017.4280

Mijan, M. A., Kim, J. Y., Moon, S. Y., Choi, S. H., Nah, S. Y., and Yang, H. J. (2019). Gintonin Enhances Proliferation, Late Stage Differentiation, and Cell Survival from Endoplasmic Reticulum Stress of Oligodendrocyte Lineage Cells. Front. Pharmacol. 10, 1211. doi:10.3389/fphar.2019.01211

Mills, S. E. E., Nicolson, K. P., and Smith, B. H. (2019). Chronic Pain: a Review of its Epidemiology and Associated Factors in Population-Based Studies. Br. J. Anaesth. 123 (2), e273-283. doi:10.1016/j.bja.2019.03.023

Mogil, J. S., Shin, Y. H., McCleskey, E. W., Kim, S. C., and Nah, S. Y. (1998). Ginsenoside Rf, a Trace Component of Ginseng Root, Produces Antinociception in Mice. Brain Res. 792, 218-228. doi:10.1016/s00068993(98)00133-4

Muley, M. M., Krustev, E., and McDougall, J. J. (2016). Preclinical Assessment of Inflammatory Pain. CNS Neurosci. Ther. 22, 88-101. doi:10.1111/cns. 12486

Nah, J. J., Hahn, J. H., Chung, S., Choi, S., Kim, Y. I., and Nah, S. Y. (2000). Effect of Ginsenosides, Active Components of Ginseng, on Capsaicin-Induced PainRelated Behavior. Neuropharmacology 39, 2180-2184. doi:10.1016/s00283908(00)00048-4

Naval, M. V., Gómez-Serranillos, M. P., Carretero, M. E., and Villar, A. M. (2007). Neuroprotective Effect of a Ginseng (Panax Ginseng) Root Extract on Astrocytes Primary Culture. J. Ethnopharmacol 112, 262-270. doi:10.1016/j. jep.2007.03.010

Nayak, D., Roth, T. L., and McGavern, D. B. (2014). Microglia Development and Function. Annu. Rev. Immunol. 32, 367-402. doi:10.1146/annurev-immunol032713-120240

Orihuela, R., McPherson, C. A., and Harry, G. J. (2016). Microglial M1/M2 Polarization and Metabolic States. Br. J. Pharmacol. 173, 649-665. doi:10. 1111/bph.13139

Park, H. Y., Lee, S. H., Lee, K. S., Yoon, H. K., Yoo, Y. C., Lee, J., et al. (2015). Ginsenoside $\operatorname{Rg} 1$ and 20(S)-Rg3 Induce IgA Production by Mouse B Cells. Immune Netw. 15, 331-336. doi:10.4110/in.2015.15.6.331

Park, J. S., Shin, J. A., Jung, J. S., Hyun, J. W., Van Le, T. K., Kim, D. H., et al. (2012). Anti-inflammatory Mechanism of Compound $\mathrm{K}$ in Activated Microglia and its Neuroprotective Effect on Experimental Stroke in Mice. J. Pharmacol. Exp. Ther. 341, 59-67. doi:10.1124/jpet.111.189035

Park, S. M., Choi, M. S., Sohn, N. W., and Shin, J. W. (2012b). 'Ginsenoside Rg(3) Attenuates Microglia Activation Following Systemic Lipopolysaccharide Treatment in Mice. Biol. Pharm. Bull. 35, 1546-1552.

Park, S. M., Choi, M. S., Sohn, N. W., and Shin, J. W. (2012a). Ginsenoside Rg3 Attenuates Microglia Activation Following Systemic Lipopolysaccharide Treatment in Mice. Biol. Pharm. Bull. 35, 1546-1552. doi:10.1248/bpb.b12-00393

Qi, L. W., Wang, C. Z., and Yuan, C. S. (2011). Isolation and Analysis of Ginseng: Advances and Challenges. Nat. Prod. Rep. 28, 467-495. doi:10.1039/ c0np00057d

Rahman, M. A., Hwang, H., Nah, S. Y., and Rhim, H. (2020). Gintonin Stimulates Autophagic Flux in Primary Cortical Astrocytes. J. Ginseng Res. 44, 67-78. doi:10.1016/j.jgr.2018.08.004

Rannou, F., Pelletier, J. P., and Martel-Pelletier, J. (2016). Efficacy and Safety of Topical NSAIDs in the Management of Osteoarthritis: Evidence from Real-Life Setting Trials and Surveys. Semin. Arthritis Rheum. 45, S18-S21. doi:10.1016/j. semarthrit.2015.11.007

Rhim, H., Kim, H., Lee, D. Y., Oh, T. H., and Nah, S. Y. (2002). Ginseng and Ginsenoside Rg3, a Newly Identified Active Ingredient of Ginseng, Modulate Ca2+ Channel Currents in Rat Sensory Neurons. Eur. J. Pharmacol. 436, 151-158. doi:10.1016/s0014-2999(01)01613-2

Rocha, H. A. C., Rocha, T. V., Nóbrega, F. J. F., Morais, L. C. S., and Diniz, M. F. F. M. (2018). Randomized Controlled Trial of Panax Ginseng in Patients with Irritable Bowel Syndrome. Revista Brasileira de Farmacognosia 28, 218-222. doi:10.1016/j.bjp.2018.02.007 
Rosenberger, D. C., Blechschmidt, V., Timmerman, H., Wolff, A., and Treede, R. D. (2020). Challenges of Neuropathic Pain: Focus on Diabetic Neuropathy. J. Neural Transm. (Vienna) 127, 589-624. doi:10.1007/s00702-020-02145-7

Ryoo, N., Rahman, M. A., Hwang, H., Ko, S. K., Nah, S. Y., Kim, H. C., et al. (2020). Ginsenoside Rk1 Is a Novel Inhibitor of NMDA Receptors in Cultured Rat Hippocampal Neurons. J. Ginseng Res. 44, 490-495. doi:10.1016/j.jgr.2019.04.002

Seth, P., Scholl, L., Rudd, R. A., and Bacon, S. (2018). Overdose Deaths Involving Opioids, Cocaine, and Psychostimulants - United States, 2015-2016. MMWR Morb Mortal Wkly Rep. 67, 349-358. doi:10.15585/mmwr.mm6712a1

Seth, R., Kuppalli, S. S., Nadav, D., Chen, G., and Gulati, A. (2021). Recent Advances in Peripheral Opioid Receptor Therapeutics. Curr. Pain Headache Rep. 25, 46. doi:10.1007/s11916-021-00951-6

Shi, D. D., Huang, Y. H., Lai, C. S. W., Dong, C. M., Ho, L. C., Li, X. Y., et al. (2019). Ginsenoside Rg1 Prevents Chemotherapy-Induced Cognitive Impairment: Associations with Microglia-Mediated Cytokines, Neuroinflammation, and Neuroplasticity. Mol. Neurobiol. 56, 5626-5642. doi:10.1007/s12035-0191474-9

Suh, H. W., Song, D. K., Huh, S. O., and Kim, Y. H. (2000). Modulatory Role of Ginsenosides Injected Intrathecally or Intracerebroventricularly in the Production of Antinociception Induced by Kappa-Opioid Receptor Agonist Administered Intracerebroventricularly in the Mouse. Planta Med. 66, 412-417. doi:10.1055/s-2000-8575

Sun, Y. Q., Shi, S. D., Zheng, Y., Liu, Y. R., Wang, S., Fu, L., et al. (2019). Analgesic Effect and Related Amino Acids Regulation of Ginsenoside Rg3 in Mouse Pain Models, 239. Life Sci. doi:10.1016/j.lfs.2019.117083

Suzuki, T., Yamamoto, A., Ohsawa, M., Motoo, Y., Mizukami, H., and Makino, T. (2017). Effect of Ninjin'yoeito and Ginseng Extracts on Oxaliplatin-Induced Neuropathies in Mice. J. Nat. Med. 71, 757-764. doi:10.1007/s11418-017-1113-6

Suzumura, A. (2013). Neuron-microglia Interaction in Neuroinflammation. Curr. Protein Pept. Sci. 14, 16-20. doi:10.2174/1389203711314010004

Tabandeh, M. R., Jafari, H., Hosseini, S. A., and Hashemitabar, M. (2015). Ginsenoside Rb1 Stimulates Adiponectin Signaling in C2C12 Muscle Cells through Up-Regulation of AdipoR1 and AdipoR2 Proteins. Pharm. Biol. 53, 125-132. doi:10.3109/13880209.2014.912237

Taherianfard, M., and Aalami, S. (2020). Effects of Pretreatment with Ginseng Extract on Dopamine D2 Receptor Analgesia. Basic Clin. Neurosci. 11, 587-593. doi:10.32598/bcn.9.10.425

Tiwari, V., Guan, Y., and Raja, S. N. (2014). Modulating the Delicate GlialNeuronal Interactions in Neuropathic Pain: Promises and Potential Caveats. Neurosci. Biobehav Rev. 45, 19-27. doi:10.1016/j.neubiorev.2014.05.002

Treede, R. D. (2018). The International Association for the Study of Pain Definition of Pain: as Valid in 2018 as in 1979, but in Need of Regularly Updated Footnotes. Pain Rep. 3, e643. doi:10.1097/PR9.0000000000000643

Tu, H., Chu, H., Guan, S., Hao, F., Xu, N., Zhao, Z., et al. (2021). The Role of the M1/M2 Microglia in the Process from Cancer Pain to Morphine Tolerance. Tissue Cell 68, 101438. doi:10.1016/j.tice.2020.101438

Um, Y., Eo, H. J., Kim, H. J., Kim, K., Jeon, K. S., and Jeong, J. B. (2020). Wild Simulated Ginseng Activates Mouse Macrophage, RAW264.7 cells through TRL2/4-dependent Activation of MAPK, NF-Kb and PI3K/AKT Pathways. J. Ethnopharmacol 263, 113218. doi:10.1016/j.jep.2020.113218

Uniyal, A., Gadepalli, A., AkhileshTiwari, V., and Tiwari, V. (2020). Underpinning the Neurobiological Intricacies Associated with Opioid Tolerance. ACS Chem. Neurosci. 11, 830-839. doi:10.1021/acschemneuro.0c00019

Uniyal, A., Thakur, V., Rani, M., TiwariGadepalli, V., Akhilesh, O., Gadepalli, A., et al. (2021). Kinesin Nanomotors Mediated Trafficking of NMDA-Loaded Cargo as A Novel Target in Chronic Pain. ACS Chem. Neurosci. 12, 2956-2963. doi:10.1021/acschemneuro.1c00319

Wang, C. Z., Yao, H., Zhang, C. F., Chen, L., Wan, J. Y., Huang, W. H., et al. (2018). American Ginseng Microbial Metabolites Attenuate DSS-Induced Colitis and Abdominal Pain. Int. Immunopharmacol 64, 246-251. doi:10.1016/j.intimp. 2018.09.005

Wang, D.-D., Kim, Y.-J., Baek, N. I., Mathiyalagan, R., Wang, C., Jin, Y., et al. (2021). Glycosyltransformation of Ginsenoside Rh2 into Two Novel Ginsenosides Using Recombinant Glycosyltransferase from Lactobacillus Rhamnosus and its In Vitro Applications. J. ginseng Res. 45, 48-57. doi:10. 1016/j.jgr.2019.11.004
Wang, L., Yuan, D., Zhang, D., Zhang, W., Liu, C., Cheng, H., et al. (2015). Ginsenoside Re Promotes Nerve Regeneration by Facilitating the Proliferation, Differentiation and Migration of Schwann Cells via the ERK- and JNKdependent Pathway in Rat Model of Sciatic Nerve Crush Injury. Cell Mol Neurobiol 35, 827-840. doi:10.1007/s10571-015-0177-7

Wei, Z., Fei, Y., Su, W., and Chen, G. (2019). Emerging Role of Schwann Cells in Neuropathic Pain: Receptors, Glial Mediators and Myelination. Front Cel Neurosci 13, 116. doi:10.3389/fncel.2019.00116

Wright, M. E., and Rizzolo, D. (2017). An Update on the Pharmacologic Management and Treatment of Neuropathic Pain. JAAPA 30, 13-17. doi:10. 1097/01.JAA.0000512228.23432.f7

Wu, C. F., Bi, X. L., Yang, J. Y., Zhan, J. Y., Dong, Y. X., Wang, J. H., et al. (2007). Differential Effects of Ginsenosides on NO and TNF-Alpha Production by LPSActivated N9 Microglia. Int. Immunopharmacol 7, 313-320. doi:10.1016/j. intimp.2006.04.021

Wu, Y., Huang, X. F., Bell, C., and Yu, Y. (2018). Ginsenoside Rb1 Improves Leptin Sensitivity in the Prefrontal Cortex in Obese Mice. CNS Neurosci. Ther. 24, 98-107. doi:10.1111/cns.12776

Xin, C., Kim, J., Quan, H., Yin, M., Jeong, S., Choi, J. I., et al. (2019). Ginsenoside Rg3 Promotes Fc Gamma Receptor-Mediated Phagocytosis of Bacteria by Macrophages via an Extracellular Signal-Regulated Kinase $1 / 2$ and P38 Mitogen-Activated Protein Kinase-dependent Mechanism. Int. Immunopharmacol 77, 105945. doi:10.1016/j.intimp.2019.105945

Xu, X., Jin, L., Jiang, T., Lu, Y., Aosai, F., Piao, H. N., et al. (2020). Ginsenoside Rh2 Attenuates Microglial Activation against Toxoplasmic Encephalitis via TLR4/NFKb Signaling Pathway. J. Ginseng Res. 44, 704-716. doi:10.1016/j.jgr.2019.06.002

Yao, F. D., Yang, J. Q., Huang, Y. C., Luo, M. P., Yang, W. J., Zhang, B., et al. (2019). Antinociceptive Effects of Ginsenoside Rb1 in a Rat Model of Cancer-Induced Bone Pain. Exp. Ther. Med. 17, 3859-3866. doi:10.3892/etm.2019.7404

Yin, H., Park, S. A., Park, S. J., and Han, S. K. (2011). Korean Red Ginseng Extract Activates Non-NMDA Glutamate and GABAA Receptors on the Substantia Gelatinosa Neurons of the Trigeminal Subnucleus Caudalis in Mice. J. Ginseng Res. 35, 219-225. doi:10.5142/jgr.2011.35.2.219

Zhang, X., Shi, M., Bjørås, M., Wang, W., Zhang, G., Han, J., et al. (2013). Ginsenoside Rd Promotes Glutamate Clearance by Up-Regulating Glial Glutamate Transporter GLT-1 via PI3K/AKT and ERK1/2 Pathways. Front. Pharmacol. 4, 152. doi:10.3389/fphar.2013.00152

Zhang, J., Li, W. B., Yuan, Q. Y., Zhou, J., Zhang, J. M., Gao, Y. F., et al. (2019). Transcriptome Analyses of the Anti-proliferative Effects of 20(S)-Ginsenoside Rh2 on HepG2 Cells. Front. Pharmacol. 10, 1331. doi:10.3389/fphar.2019.01331

Zhang, Q.-L., Li, S.-Y., and Li, P. (2019). Effects of Ginsenoside-Rg2 on Mechanical Allodynia, Heat Hyperalgeia, Depressive State of Rats with Chronic Sciatic Nerve Constriction Injury. Zhongguo Ying Yong Sheng Li Xue Za Zhi 35, 228-231. doi:10.12047/j.cjap.5763.2019.049

Zong, Y., Ai, Q. L., Zhong, L. M., Dai, J. N., Yang, P., He, Y., et al. (2012). Ginsenoside Rg1 Attenuates Lipopolysaccharide-Induced Inflammatory Responses via the Phospholipase C- $\Gamma 1$ Signaling Pathway in Murine BV-2 Microglial Cells. Curr. Med. Chem. 19, 770-779. doi:10.2174/ 092986712798992066

Conflict of Interest: The authors declare that the research was conducted in the absence of any commercial or financial relationships that could be construed as a potential conflict of interest.

Publisher's Note: All claims expressed in this article are solely those of the authors and do not necessarily represent those of their affiliated organizations, or those of the publisher, the editors and the reviewers. Any product that may be evaluated in this article, or claim that may be made by its manufacturer, is not guaranteed or endorsed by the publisher.

Copyright $\odot 2022 \mathrm{Li}$, Wei and Lu. This is an open-access article distributed under the terms of the Creative Commons Attribution License (CC BY). The use, distribution or reproduction in other forums is permitted, provided the original author $(s)$ and the copyright owner(s) are credited and that the original publication in this journal is cited, in accordance with accepted academic practice. No use, distribution or reproduction is permitted which does not comply with these terms. 